\title{
Endothelial Nitric Oxide Synthase Gene Polymorphism and the Risk of Diabetic Neuropathy in Asian Indian Patients with Type 2 Diabetes
}

\author{
Viral N Shah ${ }^{1}$, Balneek Singh Cheema ${ }^{2}$, Harbir Singh Kohli ${ }^{3}$, Rajni Sharma ${ }^{2}$, Madhu Khullar ${ }^{2}$ and Anil Bhansali ${ }^{1 *}$
}

${ }^{1}$ Department of Endocrinology, Postgraduate Institute of Medical Education and Research, Chandigarh, India

${ }^{2}$ Department of Experimental Medicine and Biotechnology, Postgraduate Institute of Medical Education and Research, Chandigarh, India

${ }^{3}$ Department of Nephrology, Postgraduate Institute of Medical Education and Research, Chandigarh, India

\begin{abstract}
Endothelial dysfunction plays a key role in the pathogenesis of diabetic vascular disease, including diabetic neuropathy (DNP). Endothelial-derived nitric oxide synthase (eNOS) gene polymorphisms affect eNOS activity and are associated with endothelial dysfunction. Studies examining association of eNOS gene polymorphism in type 2 diabetic patients (T2DM) with DNP and without DNP are lacking in Asian Indians. Thus, we investigated the association and interaction between two potentially functional single nucleotide polymorphisms (SNPs) of the eNOS gene (T-786C, G894T) and one repeat polymorphism (27VNTR) with DNP using validated PCR-RFLP assays in 1258 T2DM subjects belonging to two independent cohorts from north Indian Origin and also, we replicated our study in a $3^{\text {rd }}$ independent cohort from south Indian population having T2DM. We also measured serum Nitric Oxide (NO) levels in these subjects and examined its correlation with DNP and eNOS genotypes. We observed that eNOS genotype carrying 'aa' genotype of 27VNTR (a/b) and eNOS haplotype C-a-G and C-a-T (allele of T-786C 27 VNTR a/b and G894T) carrying 'a' allele of 27VNTR (a/b) were associated with increased risk of DNP, in all the three cohorts. We also observed lower serum NO levels in T2DM subjects (both cases and controls) carrying ba+aa genotypes. Our study suggest that 27VNTR (a/b) eNOS polymorphism carrying 'a' allele alone and in association with T-786C and G894T eNOS polymorphism is associated with increased risk of DNP in Asian Indians T2DM patients.
\end{abstract}

Keywords: Diabetic neuropathy; eNOS (endothelial nitric-oxide synthase); Gene polymorphism; Type 2 diabetes

\section{Introduction}

Diabetic peripheral neuropathy (DPN) is one of the most commonly reported microvascular complication of diabetes, affecting up to $20 \%-$ $40 \%$ of type 2 diabetic (T2DM) patients [1]. DPN is a heterogeneous disease which results from the direct damage of peripheral nerve components and of the associated vasa nervorum [1]. Also, it is a complex disorder emerging from the imbalance between multiple predisposing and protective genetic variants as well as following the interaction with environmental factors [2]. Although, chronic hyperglycemia, duration of diabetes and level of metabolic control are the main contributors to the development of DPN, however, endothelial dysfunction has been shown to be an important pathophysiologic denominator for DNP [3]. Nitric Oxide (NO) system through dramatically changed NO production and local release significantly contribute to endothelial dysfunction [4]. The process takes place by the modulation of the nitric oxide synthase (NOS) enzymes responsible for NO synthesis [5]. There are three distinct isoforms of NOS; endothelial constitutive NOS (eNOS), neuronal NOS (nNOS), and inducible NOS (iNOS) [6]. Endothelium derived NO plays a key role in the regulation of vascular tone [7], and has vaso-protective effects by scavenging superoxide radicals and suppressing platelet aggregation, leukocyte adhesion, and smooth muscle cell proliferation [7-10]. Moreover, previous studies suggest that dysfunctional endothelial nitric-oxide synthase (eNOS) might play a critical role in the pathogenic pathway leading to diabetic vascular complications including DNP [11,12]. Thus, eNOS genes became natural candidates for the study of DPN genetics. Furthermore, polymorphisms in the eNOS gene that lead to decreased eNOS expression have been found to be associated with advanced DNP suggesting that genetic polymorphisms of eNOS gene also may play a role in the $\mathrm{NO}$ abnormalities that contribute to the development and progression of DNP [13]. In comparison with the other chronic diabetes microvascular complications, data regarding the genetic background of DPN are rather scarce.

Several polymorphisms have been reported in eNOS promoter, exon and intron regions [14]. The most studied variants are the single nucleotide polymorphism (SNP) at position -786 represented by a base substitution from T to C (rs2070744), a polymorphism of eNOS in intron $4(4 \mathrm{a} / 4 \mathrm{~b})$ based on a variable 27 -base pair tandem repeat consisting of four (allele $4 \mathrm{a}$ ) or five (allele $4 \mathrm{~b}$ ) repeats, SNP at position 894 represented by a base substitution from G to T (rs1799983). In previous studies it was shown that individuals with $-786 \mathrm{C}$, 4a allele had a reduced $e N O S$ transcription rates, thus resulting in modulation of NOS3 enzymatic activity and, apparently, affecting plasma NO concentrations [15]. Study by Costacou et al. showed that G894T variant changes the $e N O S$ protein sequence, probably leading to an alteration of enzyme activity [16]. Brouet et al. also suggested that G894T variant controls the NOS3 intracellular distribution by interacting with proteins that mediate its degradation [17].

Zotova et al. (2005) analyzed the influence of eNOS polymorphisms on DPN from Caucasian patients with Type 1 Diabetic Mellitus

*Corresponding author: Anil Bhansali, Department of Endocrinology, Post Graduate Institute of Medical Education and Research, Chandigarh, India 160012 E-mail: bhansalianilendocrinology@gmail.com

Received November 12, 2012; Accepted January 12, 2013; Published January 17,2013

Citation: Shah VN, Cheema BS, Kohli HS, Sharma R, Khullar M, et al. (2013) Endothelial Nitric Oxide Synthase Gene Polymorphism and the Risk of Diabetic Neuropathy in Asian Indian Patients with Type 2 Diabetes. J Diabetes Metab 4 243. doi:10.4172/2155-6156.1000243

Copyright: $\odot 2013$ Shah VN, et al. This is an open-access article distributed unde the terms of the Creative Commons Attribution License, which permits unrestricted use, distribution, and reproduction in any medium, provided the original author and source are credited. 
Citation: Shah VN, Cheema BS, Kohli HS, Sharma R, Khullar M, et al. (2013) Endothelial Nitric Oxide Synthase Gene Polymorphism and the Risk of Diabetic Neuropathy in Asian Indian Patients with Type 2 Diabetes. J Diabetes Metab 4: 243. doi:10.4172/2155-6156.1000243

Page 2 of 7

(T1DM) genotyped for rs2070744, rs1799983 and 4b/4a polymorphism and they showed that none of these polymorphisms were associated with DPN [18]. Heltianu et al. showed that eNOS 4a/4a genotype does not represent a risk or protective factor for T1DM-DPN [14]. Other T1DM reports revealed that $894 \mathrm{GG}$ (rs1799983) homozygous patients were associated with decreased incidence of DPN [19-21]

Manea et al. showed in T2DM patients belonging to Caucasian ethnicity that frequency of $4 \mathrm{a}$ allele was significantly lower in DPN patients as compared to T2DM patients without DPN [22]. Also, Mehrab-Mohseni et al. showed in Iranian population that frequency of 4a allele was significantly lower in DPN patients as compared to T2DM patients without DPN [23]

Since, Asian Indians, an ethnically distinct population, has the leading number of diabetic patients in the world [24,25] and studies examining association of $e N O S$ gene polymorphism in type 2 diabetic patients with neuropathy and without diabetic neuropathy are lacking in Asian Indians. Moreover, there are significant genotypic differences between Indians belonging to different geographical regions [26]; thus, in the present study, we investigated the association and interaction between two potentially functional single nucleotide polymorphisms (SNPs) of the eNOS gene (T-786C,G894T) and one repeat polymorphism (27VNTR) with diabetic neuropathy in T2DM subjects belonging to two independent cohorts from north Indian Origin and also, we replicated our study in a $3^{\text {rd }}$ independent cohort from south Indian population having T2DM. We have also examined association of these SNPs with serum NO levels as the graded differences in NO generation could play a significant role in the pathophysiological condition at the microvascular level.

\section{Methods}

\section{Study population}

Two independently ascertained T2DM cohorts of North Indian origin; visiting Endocrinology and Nephrology Clinics of Postgraduate Institute of Medical Education and Research, Chandigarh, India between June 2006 and September 2007 (cohort1) and July 2009 and March 2012 (cohort 2) were studied in this study.

DNP was defined as having sensory positive symptoms such as decreased sensation, dysaesthesia, paraesthesia, numbness and muscle cramp pain in both lower legs. DNP was also investigated by assessing patients' vibration perception thresholds, using Neurothesiometer; values $\geq 25 \mathrm{~V}$ were diagnostic of DPN. None of the participants had myopathy, peripheral vascular disease or significant neurological diseases. Patients having vibration perception thresholds under $18 \mathrm{~V}$ were considered normal. Patients having bilateral values between $>18$ $\mathrm{V}$ and $<25 \mathrm{~V}$, who are considered DPN in presence of other important sign, were excluded from the study to avoid any misclassification. T2DM patients without DNP were considered as controls and healthy subjects without T2DM were not considered as control to avoid diabetes associated misclassification. Based upon this definition cohort 1 consisted of 139 patients with DNP and 356 T2DM patients without neuropathy (DM); cohort 2 had 133 patients with DNP and 342 patients had DM. Replication studies were performed in a population of 207 patients with DM and 81 DNP patients recruited from Madras Diabetes Research foundation, Chennai, South India that formed cohort 3. All the patients had same mean duration of diabetes (12.8 \pm 2.1$)$. Also, 200 healthy subjects without diabetes or other co morbidities were screened to test deviation from Hardy Weinberg Equilibrium (HWE) for these variant.
All the subjects in both groups were age, sex and ethnicity matched. The study was approved by Post Graduate Institute of Medical Education and Research, Chandigarh and Madras Diabetes Research foundation ethics committees and written consent was obtained from participating subjects for genetic testing and the genetic tests to be performed were pre-specified at the time of the study design. Their ethnicity was confirmed on the basis of language spoken and ancestral history. No regional differences in disease prevalence or allele frequency were observed between recruitment sites

\section{Genotyping}

SNPs in the eNOS gene selected for the present study are recorded in the public dbSNP database. The official gene symbol is NOS3, approved name is nitric oxide synthase 3 (endothelial cell) and location is 7q36. The SNP ID numbers and detailed sequence information are available publicly (http://www.ncbi.nlm.nih.gov/SNP/). SNPs selected for the present study were T-786C (rs 2070744), 27VNTR intron $4 \mathrm{a} / \mathrm{b}$, and G894T (rs 1799983), they were located at the promoter, Intron 4 and Exon 7 of the eNOS gene (NOS3) based on their functional nature.

Genomic DNA was extracted from peripheral blood using phenolchloroform method. Two eNOS SNPs, namely, T-786C and G894T were genotyped using the polymerase chain reaction-restriction fragment length polymorphism (PCR-RFLP). Primers for T-786C SNP were designed using Primer_3 software, whereas primers for G894T SNP were same as previously used by Shimasaki et al. [27]. The 27VNTR is characterized by presence of either four 27-bp repeats (a allele) or five 27-bp repeats (b allele) and was genotyped using PCR assay with primers as previously used by Wang et al. [28]. Details including location of SNPs in the respective genes, primer sequences, PCR conditions and restriction enzyme with product sizes are presented in table 1. Positive and negative controls were used in each genotyping run, and $5 \%$ of randomly selected samples were re-genotyped by other lab personnel with $100 \%$ concordance. The genotypes were also confirmed by sequencing of some randomly samples.

\section{Biochemical investigation}

Serum NO was measured as nitrite/nitrate levels in subjects using Griess reagent (a $1: 1$ mixture of $1 \%$ sulfanilamide in $5 \% \mathrm{H}_{3} \mathrm{PO}_{4}$ and $0.1 \% \mathrm{~N}$-(1-naphthyl)-ethylenediamine), as described by Lepoivre et al. [29].

\section{Statistical analysis}

The statistical tests were performed, using the SPSS Inc., Chicago, IL version 11.0. We tested the genotype frequencies for deviation from Hardy-Weinberg equilibrium (HWE) proportions by using HWE calculator. Using a chi-square test the deviation of genotype distribution from HWE was considered significant at $\mathrm{P}<0.05$. Discrete and continuous variables were compared between cases and controls using Pearson's $\chi^{2}$ test and unpaired $t$ test or Mann-Whitney $U$ test as appropriate. Comparison of variables between different genotypes was performed using ANOVA for normally distributed data and Kruskal-Wallis test for skewed data. Power analysis was performed using Quanto (version 1.2; http://hydra.usc.edu/gxe). Based on this analysis our sample size had the power of $86 \%$ at a small effect size (0.2) and alpha level (0.04). Haplotype frequencies were estimated in various subject groups with the help of Phase Ver 2.1 software (http:// stephenslab.uchicago.edu/software.html). We have applied fisher exact probability test for estimating level of significance of haplotype 
Citation: Shah VN, Cheema BS, Kohli HS, Sharma R, Khullar M, et al. (2013) Endothelial Nitric Oxide Synthase Gene Polymorphism and the Risk of Diabetic Neuropathy in Asian Indian Patients with Type 2 Diabetes. J Diabetes Metab 4: 243. doi:10.4172/2155-6156.1000243

Page 3 of 7

frequencies between the groups as it takes into account even the sample groups with a lower frequency, thus minimizing the error due to low frequencies. Linkage disequilibria (LD) were also estimated for the three polymorphisms in the study population, using Haploview software (http://www.broad.mit.edu/mpg/haploview/contact.php). Multivariate logistic regression was used to compute odds ratio for developing DNP by adjusting for potential confounders which include age, gender, $\mathrm{HbAlc}$, duration of diabetes, duration of hypertension, smoking, systolic blood pressure, triglyceride levels, ACEI/ARB, serum creatinine and eGFR. We excluded the potential effects of these factors on the progression of DNP by selecting subjects which had same mean duration of diabetes, were age matched, assuming that these factors are either protected or prone to developing severe microvascular complications.

\section{Results}

\section{Baseline characteristics of study participants}

Baseline characteristics of the subjects with T2DM enrolled in the three cohorts are given in table 2 . In all the three cohorts, patients with DNP had significantly higher systolic blood pressure, HbAlc, serum creatinine and lower eGFR and duration of HT as compared to DM. The mean duration of T2DM was similar in DNP and DM group.

\begin{tabular}{|c|c|c|c|c|}
\hline SNP (rs\#) & Primers & $\begin{array}{l}\text { Amplicon } \\
\text { (bp) }\end{array}$ & $\begin{array}{l}\text { Annealing temp. } \\
\left({ }^{\circ} \mathrm{C}\right) /\left[\mathrm{MgCl}_{2}\right] \\
(\mathrm{mmol})\end{array}$ & Restriction enzyme/allele size \\
\hline $\begin{array}{l}\text { T-786C } \\
\text { (rs 2070744) promoter }\end{array}$ & $\begin{array}{l}\text { P1: 5'-GCA TGC ACT CTG GCC TGA AGT G-3' } \\
\text { P2: 5'-CAG GAA GCT GCC TTC CAG TGC-3' }\end{array}$ & 223 & $61.5 / 2$ & $\begin{array}{l}\text { Mspl (Bioron)/T=162, } 61 \mathrm{bp} \\
\mathrm{C}=116,61,46 \mathrm{bp}\end{array}$ \\
\hline 27 VNTR Intron 4 & $\begin{array}{l}\text { P1: 5'-AGG CCC TAT GGT AGT GCC TTT-3' } \\
\text { P2: 5'-TCT CTT AGT GCT GTG GTC AC-3' }\end{array}$ & $393 / 420$ & $59.2 / 3.5$ & $\begin{array}{l}\text { a allele }=393 \mathrm{bp} \\
\mathrm{b} \text { allele }=420 \mathrm{bp}\end{array}$ \\
\hline $\begin{array}{l}\text { G894T } \\
\text { (rs 1799983) Exon } 7\end{array}$ & $\begin{array}{l}\text { P1: 5'-AAG GCA GGA GAC AGT GGA TGG A-3' } \\
\text { P2: 5'-CCC AGT CAA TCC CTT TGG TGC TCA-3' }\end{array}$ & 457 & $62.9 / 3$ & $\begin{array}{l}\text { Banll (New England Biolabs Inc.) } \\
\mathrm{G}=137,320 \mathrm{bp} \\
\mathrm{T}=457 \mathrm{bp}\end{array}$ \\
\hline
\end{tabular}

Table 1: Standard PCR conditions used in genotyping T-786C, G894T and 27VNTR SNPs of the eNOS gene.

\begin{tabular}{|c|c|c|c|c|c|c|c|c|c|}
\hline & \multicolumn{3}{|c|}{ Cohort 1} & \multicolumn{3}{|c|}{ Cohort 2} & \multicolumn{3}{|c|}{ Cohort 3} \\
\hline $\begin{array}{l}\text { Baseline } \\
\text { characteristics } \\
\text { (Mean } \pm \text { SD) }\end{array}$ & $\mathrm{DM}(356)$ & DNP (139) & $\begin{array}{l}P \\
\text { value }\end{array}$ & DM (342) & DNP (133) & $\begin{array}{l}P \\
\text { value }\end{array}$ & DM (207) & DNP (81) & $\begin{array}{l}P \\
\text { value }\end{array}$ \\
\hline Age (years) & $59.3 \pm 6.2$ & $60.0 \pm 5.8$ & 0.42 & $58.0 \pm 5.2$ & $57.4 \pm 6.6$ & 0.51 & $54.3 \pm 7.3$ & $53.6 \pm 4.9$ & 0.36 \\
\hline Sex M/F (n) & $\begin{array}{l}142 / 214 \\
(40 / 60)\end{array}$ & $\begin{array}{l}57 / 82 \\
(41 / 59)\end{array}$ & 0.98 & $\begin{array}{l}161 / 181 \\
(47 / 53)\end{array}$ & $\begin{array}{l}64 / 69 \\
(48 / 52)\end{array}$ & 0.81 & $\begin{array}{l}106 / 101 \\
(51 / 49)\end{array}$ & $\begin{array}{l}40 / 41 \\
(49 / 51)\end{array}$ & 0.85 \\
\hline $\begin{array}{l}\text { Duration of } \\
\text { diabetes } \\
\text { (Years) }\end{array}$ & $14.9 \pm 4.2$ & $15.2 \pm 4.8$ & 0.61 & $16.2 \pm 5.1$ & $16.7 \pm 3.9$ & 0.39 & $15.0 \pm 3.8$ & $14.8 \pm 3.6$ & 0.55 \\
\hline $\begin{array}{l}\text { Duration of } \\
\text { HTN (years) }\end{array}$ & $8.3 \pm 4.9$ & $5.6 \pm 3.6$ & $<0.001$ & $8.0 \pm 4.9$ & $5.4 \pm 4.0$ & 0.001 & $8.5 \pm 4.4$ & $5.5 \pm 4.7$ & $<0.001$ \\
\hline BMI (kg/m2) & $24.1 \pm 3.0$ & $28.2 \pm 3.3$ & 0.001 & $25.7 \pm 3.6$ & $25.8 \pm 4.0$ & 0.59 & $25.1 \pm 3.9$ & $24.7 \pm 4.0$ & 0.21 \\
\hline $\mathrm{SBP}(\mathrm{mm} \mathrm{Hg})$ & $136.2 \pm 14.6$ & $146.3 \pm 18.0$ & $<0.001$ & $130.2 \pm 12.3$ & $142.6 \pm 15.2$ & 0.001 & $128.9 \pm 15.6$ & $145.5 \pm 18.0$ & 0.001 \\
\hline $\mathrm{DBP}(\mathrm{mm} \mathrm{Hg})$ & $83.3 \pm 7.6$ & $88.9 \pm 8.9$ & 0.001 & $81.0 \pm 7.4$ & $81.5 \pm 7.4$ & 0.25 & $76.9 \pm 10.2$ & $84.6 \pm 10.0$ & $<0.001$ \\
\hline HbA1c (\%) & $8.1 \pm 2.3$ & $8.9 \pm 1.4$ & $<0.001$ & $8.2 \pm 1.6$ & $8.9 \pm 2.0$ & 0.002 & $8.2 \pm 1.9$ & $8.9 \pm 1.6$ & $<0.001$ \\
\hline $\begin{array}{l}\text { S.creat } \\
\text { (mg/dl) }\end{array}$ & $1.18 \pm 0.15$ & $4.12 \pm 2.01$ & $<0.001$ & $1.5 \pm 0.3$ & $4.0 \pm 1.3$ & $<0.001$ & $1.0 \pm 0.3$ & $4.6 \pm 1.2$ & $<0.001$ \\
\hline eGFR (ml/min) & $98.4 \pm 18.6$ & $58.7 \pm 33.7$ & 0.002 & $98.3 \pm 16.3$ & $57.3 \pm 35.5$ & 0.003 & $98.6 \pm 17.7$ & $54.8 \pm 18.0$ & 0.002 \\
\hline TC & $260.1 \pm 42.3$ & $255.3 \pm 46.3$ & 0.85 & $264.3 \pm 40.6$ & $253.6 \pm 48.8$ & 0.77 & $251.1 \pm 44.0$ & $255.9 \pm 36.6$ & 0.89 \\
\hline TG $(m g \%)$ & $208.3 \pm 41.2$ & $204.6 \pm 32.8$ & 0.79 & $207.4 \pm 40.2$ & $204.3 \pm 30.1$ & 0.65 & $203.6 \pm 42.3$ & $207.7 \pm 31.0$ & 0.75 \\
\hline HDL (mg\%) & $61.3 \pm 14.4$ & $64.4 \pm 14.0$ & 0.46 & $62.1 \pm 13.8$ & $63.7 \pm 11.8$ & 0.35 & $61.7 \pm 12.5$ & $62.7 \pm 13.5$ & 0.71 \\
\hline LDL-C ( mg/dl) & $98.6 \pm 30.1$ & $102.3 \pm 41.6$ & 0.66 & $97.6 \pm 29.0$ & $101.3 \pm 27.7$ & 0.36 & $97.1 \pm 26.5$ & $101.0 \pm 26.3$ & 0.65 \\
\hline
\end{tabular}

M: Male; F: Female; BMI: Body Mass Index; HTN: Hypertension; SBP: Systolic Blood Pressure; DBP: Diastolic Blood Pressure; S.creat: Serum Creatinine; eGFR: Estimated Glomerular Filtration Rate by MDRD Formula; TC: Total Cholesterol; TG: Triglyceride; LDL-C: Low Density Lipoprotein Cholesterol; HDL-C: High Density Lipoprotein Cholesterol $(p<0.05$ is significant).

${ }^{*} \mathrm{P}$ value is after adjustment for multivariate regression analysis. Values are mean $\pm \mathrm{SD}$; unless indicated otherwise

Table 2: Baseline characteristics of study subjects. 
Citation: Shah VN, Cheema BS, Kohli HS, Sharma R, Khullar M, et al. (2013) Endothelial Nitric Oxide Synthase Gene Polymorphism and the Risk of Diabetic Neuropathy in Asian Indian Patients with Type 2 Diabetes. J Diabetes Metab 4: 243. doi:10.4172/2155-6156.1000243

Page 4 of 7

\section{Genetic analysis}

All the polymorphisms examined in the present study were in Hardy-Weinberg equilibrium in all the three Groups from all the three cohorts ( $p>0.05$ ) except T-786C (rs 2070744) where significant deviation from HWE of genotype distribution was observed in the present population. Significant deviation from Hardy Weinberg equilibrium was also observed in 200 healthy subjects without diabetes or other co morbidities in T-786C (rs 2070744). Genotype frequencies of the T-786C (rs 2070744), G894T (rs 1799983) and 27VNTR (a/b) SNPs are given in table 3. In all the three cohorts, the frequency of ba, aa and ba+aa genotype of $27 \operatorname{VNTR}(\mathrm{a} / \mathrm{b})$ was found to be significantly higher as compared to bb genotype in Groups compared with and without DNP, with a high-risk ratio in the DNP Group as compared to DM Group. No association with increased or decreased risk of DNP was observed with respect to other eNOS genotypes (T-786C, G894T) in any of the three cohorts.

\section{Biochemical analysis}

In all the three cohorts, serum NO levels were significantly decreased in DNP Group as compared to DM Group. We observed lower mean NO levels in T2DM subjects (both with and without DNP) carrying ba+aa Genotype of $27 \operatorname{VNTR}(\mathrm{a} / \mathrm{b})$ as compared to bb genotype. No significant difference in serum NO levels was observed with respect to other eNOS genotypes i.e. T-786C, G894T (Table 4) in any of the three cohorts.

\section{Haplotype analysis}

In all the three cohorts, Frequency of haplotypes C-a-G and C-a-T (allele of T-786C, 27VNTR a/b and G894T) carrying 'a' allele of 27VNTR $(\mathrm{a} / \mathrm{b})$ and $\mathrm{C}$ allele of T-786C was significantly higher in patients having DNP as compared to patients having DM and these haplotypes were associated with a increased risk of DNP. No association with increased or decreased risk of DNP was observed with respect to other six eNOS haplotypes (Table 5) in any of the three cohorts.

\begin{tabular}{|c|c|c|c|c|c|c|c|c|c|c|}
\hline & & \multicolumn{3}{|c|}{ Cohort 1} & \multicolumn{3}{|c|}{ Cohort 2} & \multicolumn{3}{|c|}{ Cohort 3} \\
\hline Polymorphisms & $\begin{array}{l}\text { Healthy } \\
\text { Controls } \\
(n=200)\end{array}$ & $\mathrm{DM}(356)$ & DNP (139) & $\begin{array}{l}\text { OR }(95 \% \mathrm{Cl}) \\
P\end{array}$ & DM (342) & DNP (133) & $\begin{array}{l}\text { OR }(95 \% \mathrm{Cl}) \\
\mathrm{P}\end{array}$ & DM (207) & DNP (81) & $\begin{array}{l}\text { OR }(95 \% \mathrm{Cl}) \\
\mathrm{P}\end{array}$ \\
\hline $\begin{array}{l}\text { T-786C } \\
\text { (rs 2070744) }\end{array}$ & $\begin{array}{l}\mathrm{TT}=131(0.66) \\
\mathrm{TC}=67(0.33) \\
\mathrm{CC}=2(0.01) \\
\\
\mathrm{TC}+\mathrm{CC}=69 \\
(0.34) \\
\\
\mathrm{T}=329(0.82) \\
\mathrm{C}=71(0.18)\end{array}$ & $\begin{array}{l}\mathrm{TT}=199(0.56) \\
\mathrm{TC}=121(0.34) \\
\mathrm{CC}=36 \\
(0.10) \\
\\
\mathrm{TC}+\mathrm{CC}=157 \\
(0.44) \\
\\
\mathrm{T}=519(0.73) \\
\mathrm{C}=193(0.27)\end{array}$ & $\begin{array}{l}\mathrm{TT}=72 \\
(0.52) \\
\mathrm{TC}=50(0.36) \\
\mathrm{CC}=17 \\
(0.12) \\
\mathrm{TC}+\mathrm{CC}=67 \\
(0.48) \backslash \\
\mathrm{T}=194(0.70) \\
\mathrm{C}=84(0.30)\end{array}$ & $\begin{array}{l}1.22(0.76- \\
1.89) 0.79 \\
\\
1.30(0.65- \\
2.90) 0.72 \\
\\
1.11(0.70- \\
1.90) 0.61 \\
\\
1.16(0.86- \\
1.58) 0.37\end{array}$ & $\begin{array}{l}\mathrm{TT}=171(0.50) \\
\mathrm{TC}=130(0.38) \\
\mathrm{CC}=41 \quad(0.12) \\
\mathrm{TC}+\mathrm{CC}=166 \\
(0.50) \\
\mathrm{T}=472(0.69) \\
\mathrm{C}=212(0.31)\end{array}$ & $\begin{array}{l}\mathrm{TT}=66 \\
(0.50) \\
\mathrm{TC}=52 \quad(0.39) \\
\\
\mathrm{CC}=15 \\
(0.11) \\
\\
\mathrm{TC}+\mathrm{CC}=67 \\
(0.50) \\
\\
\mathrm{T}=184(0.69) \\
\mathrm{C}=82(0.31)\end{array}$ & $\begin{array}{l}1.12(0.80- \\
1.55) 0.98 \\
1.15 \quad(0.66- \\
1.90) 1.02 \\
\\
1.12(0.81- \\
1.55) 0.80 \\
\\
0.99(0.73- \\
1.35) 1\end{array}$ & $\begin{array}{l}\mathrm{TT}=107(0.52) \\
\mathrm{TC}=75(0.36) \\
\mathrm{CC}=25 \\
(0.12) \\
\mathrm{TC}+\mathrm{CC}=100 \\
(0.48) \\
\mathrm{T}=289(0.70) \\
\mathrm{C}=125(0.30)\end{array}$ & $\begin{array}{l}\mathrm{TT}=40(0.49) \\
\mathrm{TC}=31(0.39) \\
\mathrm{CC}=10(0.12) \\
\mathrm{TC}+\mathrm{CC}=41 \\
(0.51) \\
\\
\mathrm{T}=111(0.69) \\
\mathrm{C}=51(0.31)\end{array}$ & $\begin{array}{l}1.22(0.85- \\
1.45) 0.90 \\
1.11 \quad(0.62- \\
1.98) 0.94 \\
1.20(0.83- \\
1.61) 1.02 \\
1.06(0.72- \\
1.57) 0.84\end{array}$ \\
\hline $\begin{array}{l}\text { G894T } \\
\text { (rs 1799983) }\end{array}$ & $\begin{array}{l}\mathrm{GG}=102 \\
(0.51) \\
\mathrm{GT}=80(0.40) \\
\mathrm{TT}=18(0.09) \\
\mathrm{GT}+\mathrm{TT}=98 \\
(0.49) \\
\mathrm{G}=284(0.71) \\
\mathrm{T}=116(0.29)\end{array}$ & $\begin{array}{l}\mathrm{GG}=156(0.44) \\
\mathrm{GT}=164(0.46) \\
\mathrm{TT}=36 \\
(0.10) \\
\mathrm{GT}+\mathrm{TT}=200 \\
(0.56) \\
\mathrm{G}=476(0.67) \\
\mathrm{T}=236(0.33)\end{array}$ & $\begin{array}{l}\mathrm{GG}=65 \\
(0.47) \\
\mathrm{GT}=60 \\
(0.43) \\
\mathrm{TT}=14 \\
(0.10) \\
\mathrm{GT}+\mathrm{TT}=74 \\
(0.53) \\
\mathrm{G}=190(0.68) \\
\mathrm{T}=88(0.32)\end{array}$ & $\begin{array}{l}0.94(0.59- \\
1.60) 0.79 \\
\\
0.94(0.42- \\
2 . .22) 0.89 \\
\\
0.95(0.59- \\
1.52) 0.78 \\
\\
0.93(0.69- \\
1.26) 0.71\end{array}$ & $\begin{array}{l}\mathrm{GG}=157(0.46) \\
\mathrm{GT}=144(0.42) \\
\mathrm{TT}=41 \\
(0.12) \\
\mathrm{GT}+\mathrm{TT}=185 \\
(0.54) \\
\mathrm{G}=458(0.67) \\
\mathrm{T}=226(0.33)\end{array}$ & $\begin{array}{l}\mathrm{GG}=63 \\
(0.47) \\
\mathrm{GT}=57 \\
(0.43) \\
\mathrm{TT}=13 \\
(0.10) \\
\mathrm{GT}+\mathrm{TT}=70 \\
(0.53) \\
\mathrm{G}=183(0.69) \\
\mathrm{T}=83(0.31)\end{array}$ & $\begin{array}{l}1.08(0.72- \\
1.52) 1.05 \\
0.80(0.41- \\
1.52) 0.62 \\
1.20(0.77- \\
1 . .48) 0.95 \\
\\
0.92(0.68- \\
1.25) 0.64\end{array}$ & $\begin{array}{l}\mathrm{GG}=97 \\
(0.47) \\
\mathrm{GT}=87 \quad(0.42) \\
\mathrm{TT}=23 \\
(0.11) \\
\mathrm{GT}+\mathrm{TT}=110 \\
(0.53) \\
\mathrm{G}=281(0.68) \\
\mathrm{T}=133(0.32)\end{array}$ & $\begin{array}{l}\mathrm{GG}=39 \\
(0.48) \\
\mathrm{GT}=34 \\
(0.42) \\
\mathrm{TT}=8 \\
(0.10) \\
\mathrm{GT}+\mathrm{TT}=42 \\
(0.52) \\
\mathrm{G}=112(0.69) \\
\mathrm{T}=50(0.31)\end{array}$ & $\begin{array}{l}1.03 \quad(0.75- \\
1.51) 0.89 \\
\\
0.97(0.42- \\
1.51) 0.87 \\
0.90(0.71- \\
.1 .42) 0.95 \\
\\
0.94(0.64- \\
1.40) 0.84\end{array}$ \\
\hline 27 VNTR & $\begin{array}{l}a a=144(0.72) \\
b a+a a=193 \\
(0.97) \\
\\
b=63(0.16) \\
a=337(0.84)\end{array}$ & $\begin{array}{l}b b=217(0.61) \\
b a^{*}=121 \\
(0.34) \\
a a^{*}=18 \\
(0.05) \\
\\
b a+a a^{*}=139 \\
(0.39) \\
b=555(0.78) \\
a^{*}=157(0.22)\end{array}$ & $\begin{array}{l}\mathrm{bb}=61 \\
(0.44) \\
\mathrm{ba}^{*}=63 \\
(0.45) \\
\\
a a^{*}=15 \\
(0.11) \\
\\
b a+a a^{*}=78 \\
(0.56) \\
b=185(0.67) \\
a^{*}=93(0.33)\end{array}$ & $\begin{array}{l}1.85(1.22- \\
2.81) 0.005 \\
2.96(1.41- \\
3.22) 0.006 \\
2.00(1.34- \\
2.97) 0.008 \\
\\
1.77(1.31- \\
2.41) \\
0.003\end{array}$ & $\begin{array}{l}b b=202(0.59) \\
b a^{*}=121 \\
(0.35) \\
\\
a a^{*}=21 \\
(0.06) \\
\\
b a+a a^{*}=142 \\
(0.41) \\
\\
b=523(0.76) \\
a^{*}=161(0.24)\end{array}$ & $\begin{array}{l}\mathrm{bb}=59 \\
(0.44) \\
\mathrm{ba}^{*}=57 \\
(0.43) \\
\\
\mathrm{a} a^{*}=17 \\
(0.13) \\
\\
\mathrm{ba}+\mathrm{aa} a^{*}=74 \\
(0.56) \\
\mathrm{b}=175(0.66) \\
\mathrm{a}^{*}=91(0.34)\end{array}$ & $\begin{array}{l}1.61(1.05- \\
2.47) 0.04 \\
2.77(1.37- \\
3.59) 0.006 \\
1.78(1.19- \\
2.67) 0.006 \\
1.69(1.24- \\
2.30) 0.001\end{array}$ & $\begin{array}{l}b b=117(0.57) \\
b a^{*}=75 \\
(0.36) \\
\\
a a^{*}=14 \\
(0.07) \\
\\
b a+a a^{*}=89 \\
(0.41) \\
b=310(0.75) \\
a^{*}=104(0.25)\end{array}$ & $\begin{array}{l}b b=37 \\
(0.46) \\
b a^{*}=33 \\
(0.41) \\
\\
a a^{*}=11 \\
(0.13) \\
\\
b a+a a^{*}=44 \\
(0.54) \\
b=107(0.66) \\
a^{*}=55(0.34)\end{array}$ & $\begin{array}{l}1.39(1.07- \\
2.42) 0.03 \\
\\
2.48(1.84- \\
3.92) 0.006 \\
\\
1.56(1.10- \\
2.61) 0.62 \\
1.53(1.13- \\
2.27) 0.004\end{array}$ \\
\hline
\end{tabular}

${ }^{*} \mathrm{P}<0.05 \mathrm{P}$ values for the model are adjusted for potential confounders which include age, gender, HbA1c, duration of diabetes, duration of hypertension, smoking, systolic blood pressure, triglyceride levels, ACEI/ARB, serum creatinine and eGFR

Table 3: Genotype and Allele frequencies of eNOS variants in T2DM patients (DM vs. DNP). 
Citation: Shah VN, Cheema BS, Kohli HS, Sharma R, Khullar M, et al. (2013) Endothelial Nitric Oxide Synthase Gene Polymorphism and the Risk of Diabetic Neuropathy in Asian Indian Patients with Type 2 Diabetes. J Diabetes Metab 4: 243. doi:10.4172/2155-6156.1000243

Page 5 of 7

\begin{tabular}{|c|c|c|c|c|c|c|c|c|c|c|c|c|c|c|c|c|c|c|c|c|c|c|c|c|c|c|c|}
\hline \multirow{3}{*}{$\begin{array}{l}\text { NO } \\
\text { levels } \\
\text { (nmol/l) }\end{array}$} & \multicolumn{9}{|c|}{ Cohort 1} & \multicolumn{9}{|c|}{ Cohort 2} & \multicolumn{9}{|c|}{ Cohort 1} \\
\hline & \multicolumn{3}{|c|}{ T-786C } & \multicolumn{3}{|c|}{ 27VNTR } & \multicolumn{3}{|c|}{ G894T } & \multicolumn{3}{|c|}{ T-786C } & \multicolumn{3}{|c|}{ 27VNTR } & \multicolumn{3}{|c|}{ G894T } & \multicolumn{3}{|c|}{$T-786 C$} & \multicolumn{3}{|c|}{ 27VNTR } & \multicolumn{3}{|c|}{ G894T } \\
\hline & TT & $\mathrm{TC}+\mathrm{CC}$ & $P$ & $\mathrm{bb}$ & ba+aa & $P^{*}$ & TT & $\mathrm{TC}+\mathrm{CC}$ & $P$ & TT & $\mathrm{TC}+\mathrm{CC}$ & $P$ & $\mathrm{bb}$ & $b a+a a$ & $P^{*}$ & TT & $\mathrm{TC}+\mathrm{CC}$ & P & TT & $\mathrm{TC}+\mathrm{CC}$ & $P$ & $\mathrm{bb}$ & ba+aa & $\mathrm{P}^{*}$ & TT & $\mathrm{TC}+\mathrm{CC}$ & $P$ \\
\hline DM & $\begin{array}{l}0.62 \\
\pm \\
0.12\end{array}$ & $\begin{array}{l}0.64 \pm \\
0.14\end{array}$ & 0.15 & $\begin{array}{l}0.75 \\
\pm \\
0.18\end{array}$ & $\begin{array}{l}0.50 \pm \\
0.17\end{array}$ & 0.03 & $\begin{array}{l}0.63 \\
\pm \\
0.18\end{array}$ & $\begin{array}{l}0.65 \pm \\
0.20\end{array}$ & 0.22 & $\begin{array}{l}0.60 \\
\pm \\
0.11\end{array}$ & $\begin{array}{l}0.62 \pm \\
0.10\end{array}$ & 0.14 & $\begin{array}{l}0.76 \\
\pm \\
0.12\end{array}$ & $\begin{array}{l}0.52 \pm \\
0.15\end{array}$ & 0.02 & $\begin{array}{l}0.66 \\
\pm \\
0.19\end{array}$ & $\begin{array}{l}0.67 \pm \\
0.14\end{array}$ & 0.18 & \begin{tabular}{|l}
0.64 \\
\pm \\
0.14
\end{tabular} & $\begin{array}{l}0.67 \pm \\
0.15\end{array}$ & 0.14 & $\begin{array}{l}0.70 \\
\pm \\
0.11\end{array}$ & $\begin{array}{l}0.55 \pm \\
0.14\end{array}$ & 0.01 & $\begin{array}{l}0.65 \\
\pm \\
0.15\end{array}$ & $\begin{array}{l}0.63 \pm \\
0.10\end{array}$ & 0.20 \\
\hline DNP & $\begin{array}{l}0.33 \\
\pm \\
0.13\end{array}$ & $\begin{array}{l}0.35 \pm \\
0.15\end{array}$ & 0.12 & \begin{tabular}{|l}
0.42 \\
\pm \\
0.17
\end{tabular} & $\begin{array}{l}0.28 \pm \\
0.17\end{array}$ & 0.04 & $\begin{array}{l}0.38 \\
\pm \\
0.14\end{array}$ & $\begin{array}{l}0.36 \pm \\
0.12\end{array}$ & 0.20 & $\begin{array}{l}0.32 \\
\pm \\
0.11\end{array}$ & $\begin{array}{l}0.33 \pm \\
0.12\end{array}$ & 0.10 & $\begin{array}{l}0.43 \\
\pm \\
0.15\end{array}$ & $\begin{array}{l}0.29 \pm \\
0.17\end{array}$ & 0.03 & $\begin{array}{l}0.40 \\
\pm \\
0.12\end{array}$ & $\begin{array}{l}0.37 \pm \\
0.14\end{array}$ & 0.10 & $\begin{array}{l}0.35 \\
\pm \\
0.14\end{array}$ & $\begin{array}{l}0.36 \pm \\
0.14\end{array}$ & 0.11 & $\begin{array}{l}0.40 \\
\pm \\
0.12\end{array}$ & $\begin{array}{l}0.30 \pm \\
0.16\end{array}$ & 0.02 & $\begin{array}{l}0.37 \\
\pm \\
0.16\end{array}$ & $\begin{array}{l}0.38 \pm \\
0.14\end{array}$ & 0.18 \\
\hline
\end{tabular}

Values are mean \pm standard deviation, ${ }^{*} \mathrm{P}<0.05$ is significant

Table 4: eNOS genotypes and NO levels.

\begin{tabular}{|c|c|c|c|c|c|c|c|c|c|}
\hline \multirow[b]{2}{*}{ Haplotype ${ }^{a}$} & \multicolumn{3}{|c|}{ Cohort 1} & \multicolumn{3}{|c|}{ Cohort 2} & \multicolumn{3}{|c|}{ Cohort 3} \\
\hline & DNP (139) & $\mathrm{DM}(356)$ & OR (95\%Cl) P & DNP (133) & DM (342) & OR (95\%Cl) P & DNP (81) & DM (207) & OR $(95 \% \mathrm{Cl}) \mathrm{P}$ \\
\hline T-b-G & $17(0.12)$ & $46(0.13)$ & Reference & $15(0.11)$ & $44(0.13)$ & Reference & $10(0.12)$ & $29(0.14)$ & Reference \\
\hline T-b-T & $18(0.14)$ & $53(0.15)$ & $\begin{array}{l}1.09(0.82-1.55) \\
0.88\end{array}$ & $16(0.12)$ & $48(0.14)$ & $\begin{array}{l}0.92(0.70-1.32) \\
0.88\end{array}$ & $11(0.14)$ & $33(0.16)$ & $\begin{array}{l}0.99(0.68-1.36) \\
0.22\end{array}$ \\
\hline C-b-G & $13(0.09)$ & $39(0.11)$ & $\begin{array}{l}1.23(0.99-1.59) \\
0.21\end{array}$ & $15(0.11)$ & $44(0.13)$ & $\begin{array}{l}1.13(1.02-1.80) \\
0.12\end{array}$ & $8(0.10)$ & $25(0.12)$ & $\begin{array}{l}1.25(0.92-1.77) \\
0.31\end{array}$ \\
\hline T-a-G & $13(0.09)$ & $39(0.11)$ & $\begin{array}{l}0.69(0.30-1.37) \\
0.55\end{array}$ & $13(0.10)$ & $41(0.12)$ & $\begin{array}{l}1.22(0.79-1.54) \\
0.91\end{array}$ & $8(0.10)$ & $27(0.13)$ & $\begin{array}{l}0.93(0.55-1.34) \\
0.77\end{array}$ \\
\hline C-b-T & $22(0.16)$ & $61(0.17)$ & $\begin{array}{l}0.69(0.35-1.44) \\
0.67\end{array}$ & $21(0.17)$ & $65(0.19)$ & $\begin{array}{l}0.79(0.44-1.14) \\
0.37\end{array}$ & $12(0.15)$ & $35(0.17)$ & $\begin{array}{l}0.77(0.42-1.11) \\
0.45\end{array}$ \\
\hline T-a-T & $24(0.17)$ & $68(0.19)$ & $\begin{array}{l}1.12(0.88-1.81) \\
0.13\end{array}$ & $23(0.17)$ & $58(0.17)$ & $\begin{array}{l}1.55(1.21-1.92) \\
0.12\end{array}$ & $13(0.16)$ & $38(0.18)$ & $\begin{array}{l}0.99(0.59-1.55) \\
1.02\end{array}$ \\
\hline C-a-G* & $17(0.12)$ & $25(0.07)$ & $\begin{array}{l}1.55(1.22-2.80) \\
0.01\end{array}$ & $15(0.11)$ & $21(0.06)$ & $\begin{array}{l}1.37(1.20-2.94) \\
0.01\end{array}$ & $10(0.12)$ & $10(0.05)$ & $\begin{array}{l}1.65(1.42-2.91) \\
0.01\end{array}$ \\
\hline$C-a-T^{*}$ & $15(0.11)$ & $25(0.07)$ & $\begin{array}{l}1.71(1.31-2.96) \\
0.008\end{array}$ & $15(0.11)$ & $21(0.06)$ & $\begin{array}{l}1.71(1.47-2.98) \\
0.009\end{array}$ & $9(0.11)$ & $10(0.05)$ & $\begin{array}{l}1.77(1.57-2.94) \\
0.007\end{array}$ \\
\hline
\end{tabular}

a Order of SNPs in eNOS haplotypes: T-786C, a 27VNTR b and G894T. *P<0.05

Table 5: eNOS gene Haplotype Frequency Distribution (DM vs. DNP).

\begin{tabular}{|c|c|c|c|c|c|c|c|c|c|c|c|c|c|c|}
\hline \multicolumn{5}{|c|}{ Cohort 1} & \multicolumn{5}{|c|}{ Cohort 2} & \multicolumn{5}{|c|}{ Cohort 3} \\
\hline Variant 1 & Variant 2 & D' & LOD & $r^{2}$ & Variant 1 & Variant 2 & D' & LOD & $r^{2}$ & Variant 1 & Variant 2 & D' & LOD & $r^{2}$ \\
\hline T-786C & 27VNTR & 0.02 & 0.04 & 0.00 & T-786C & 27VNTR & 0.03 & 0.06 & 0.01 & T-786C & 27VNTR & 0.02 & 0.07 & 0.00 \\
\hline T-786C & G894T & 0.10 & 0.14 & 0.01 & T-786C & G894T & 0.09 & 0.22 & 0.01 & T-786C & G894T & 0.08 & 0.20 & 0.01 \\
\hline 27VNTR & G894T & 0.03 & 0.04 & 0.00 & 27VNTR & G894T & 0.02 & 0.03 & 0.00 & 27VNTR & G894T & 0.02 & 0.02 & 0.01 \\
\hline
\end{tabular}

Table 6: Measures of LD (D', LOD, and $\left.r^{2}\right)$, observed as a pair-wise comparison for the three polymorphisms of the eNOS gene.

\section{Linkage Disequilibria (LD) analysis}

LD values were generated to look for association among the three studied polymorphisms (Figure 1). A pair-wise comparison of the polymorphisms, depicting the LD measures is represented in table 6. In all the three cohorts, no significant LD was observed among the polymorphisms (T-786C, 27VNTR, G894T) (Table 6).

\section{Discussion}

We observed that eNOS genotype carrying 'aa', 'ba', 'ba+aa' genotype of 27VNTR $(\mathrm{a} / \mathrm{b})$, 'a' allele of 27VNTR $(\mathrm{a} / \mathrm{b})$ and $e N O S$ haplotype C-a-G and C-a-T (allele of T-786C, 27VNTR a/b and G894T) carrying 'a' allele of 27VNTR (a/b) and C allele of T-786C were associated with increased risk of DNP. Moreover, the present study fulfills most of the criteria of a good genetic association study, as suggested by Hattersley and McCarthy [30]. All the study population was in HWE, however, significant deviation from HWE of genotype distribution in the present population in T-786C gene polymorphism may be due to moderate population size. High frequency of mutation occurring at the specific loci can also cause deviation from equilibrium of genotype distribution in the present population. Moreover, we excluded the possibility of a typing error (LOD Score $>$ zero). Also, we screened one control group composed by 200 healthy subjects without diabetes or other co morbidities to help us elucidate the plausible fact that Asian Indians are not in HWE for this variant, moreover, as we used the same conditions for the PCR-RFLP method as described by Ahluwalia et al. [31] and genotype distribution in their study was also not in HWE. Moreover, positive association observed between eNOS SNPs and DNP was not due to chance, as this association was replicated in three independent cohorts and persisted even after the influence of confounding factors was corrected, also the sample size was predetermined for the three polymorphisms to avoid type 1 error $[32,33]$. Also, our study has the statistical power to detect influence of these polymorphisms on the course of DNP.

The mechanisms responsible for the association between the eNOS gene polymorphisms and the risk of DNP are not completely known. We observed lower serum NO levels in T2DM subjects (both cases and controls) carrying ba+aa genotypes. The graded differences in NO generation could play a significant role in the pathophysiological 
Citation: Shah VN, Cheema BS, Kohli HS, Sharma R, Khullar M, et al. (2013) Endothelial Nitric Oxide Synthase Gene Polymorphism and the Risk of Diabetic Neuropathy in Asian Indian Patients with Type 2 Diabetes. J Diabetes Metab 4: 243. doi:10.4172/2155-6156.1000243

Page 6 of 7

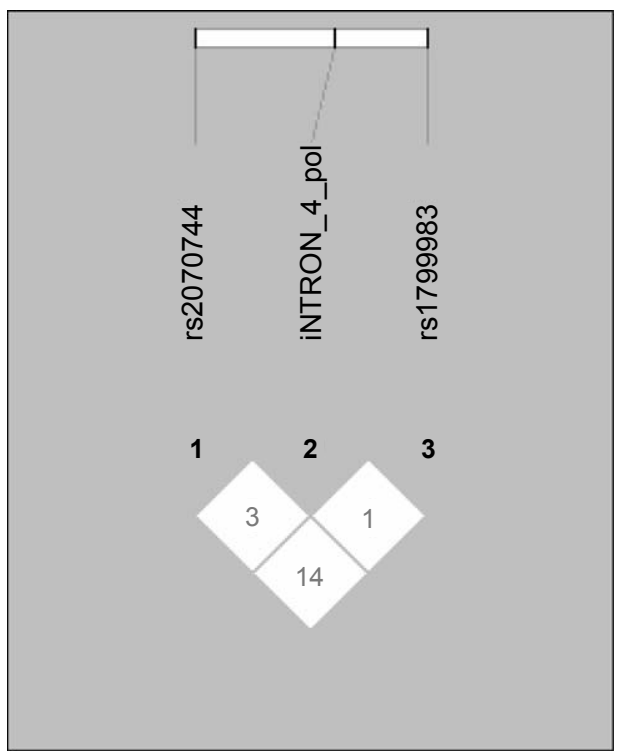

Figure 1: LD Plot showing the position of the three polymorphisms and pairwise D' values observed (within the diagonal boxes).

condition at the microvascular level. Lower NO level could accelerate the progression of microvascular complications of diabetes, presumably through mechanisms, such as increased vascular tone and enhancement of angiotensin II effects [34,35]. aa 27VNTR bb polymorphism is known to affect the activity of eNOS and has been found to be associated with a significant reduction in the eNOS gene promoter activity [36]; lower eNOS mRNA and serum nitrite/nitrate levels have been found in individuals with the 27VNTR aa variant in some studies [37-39] and 27VNTR aa variant has also been found to be associated with a significant reduction in the eNOS gene promoter activity [36]. However, we were aware that serum NO is not necessarily correlated with $e N O S$ activity due to the fact of presence of three NOS activities that may contribute in the serum NO level; eNOS, inducible NOS (iNOS) and neuronal NOS (nNOS). In fact, NO level is affected more by the iNOS activity which might be enhanced in systemic inflammation associated with the disease. This might explain why this study contradicts our previous work performed with the participation of the same cohort of patients [40], where in the mean NO levels in T2DM subjects were significantly higher in carriers of the "ba+aa" genotype of 27VNTR gene compared to "bb" genotype which contradicts the principal that SNP may affect the enzyme in one direction only or it may be nonfunctional.

Our study was in disagreement with two previous studies by Manea et al. [22] and Mehrab-Mohseni et al. [23] from Caucasian and Iranian population respectively who observed significantly higher proportion of $4 \mathrm{a}$ allele carriers in free complications patients than in DNP patients. However, Asian Indians, an ethnically distinct population, has the leading number of diabetic patients in the world and there are significant genotypic differences between Asian Indians and other ethnic groups belonging to different geographical regions [26]. Nevertheless, additional data to confirm these findings are needed. Moreover, we maintained stringent criteria to define DNP, no regional differences in disease prevalence or allele frequency were observed between recruitment sites. The relatively medium size of our case-control study is a limitation that could introduce type 1 errors; however, very few investigators have access to a large sample size.
Haplotype analyses of $e N O S$ gene may not reliable due to the small number of subjects in several haplotypes and positive associations observed in the interaction models may be due to combination of SNPs showing positive associations. Also, we were aware that combining the three cohorts would increase the statistical power of the study; however, we were unable to merge the three cohorts as they were independent cohorts enrolled at different time interval.

\section{References}

1. Holzer SE, Camerota A, Martens L, Cuerdon T, Crystal-Peters J, et al. (1998) Costs and duration of care for lower extremity ulcers in patients with diabetes. Clin Ther 20: 169-181.

2. Burdon KP, Langefeld CD, Wagenknecht LE, Carr JJ, Freedman BI, et al. (2006) Association analysis of genes in the renin-angiotensin system with subclinical cardiovascular disease in families with type 2 diabetes mellitus: the Diabetes Heart Study. Diabet Med 23: 228-234.

3. Moncada S, Higgs A (1993) The L-arginine-nitric oxide pathway. N Engl J Med 329: 2002-2012.

4. Loscalzo J, Welch G (1995) Nitric oxide and its role in the cardiovascular system. Prog Cardiovasc Dis 38: 87-104.

5. Cooke JP, Tsao PS (1994) Is NO an endogenous antiatherogenic molecule? Arterioscler Thromb 14: 653-655

6. Star RA (1997) Intrarenal localization of nitric oxide synthase isoforms and soluble guanylyl cyclase. Clin Exp Pharmacol Physiol 24: 607-610.

7. Moncada S, Palmer RM, Higgs EA (1991) Nitric oxide: physiology, pathophysiology, and pharmacology. Pharmacol Rev 43: 109-142.

8. Schmidt HH, Walter U (1994) NO at work. Cell 78: 919-925.

9. Lefer AM (1997) Nitric oxide: nature's naturally occurring leukocyte inhibitor Circulation 95: 553-554.

10. Garg UC, Hassid A (1989) Nitric oxide-generating vasodilators and 8-bromocyclic guanosine monophosphate inhibit mitogenesis and proliferation of cultured rat vascular smooth muscle cells. J Clin Invest 83: 1774-1777.

11. Prabhakar SS (2004) Role of nitric oxide in diabetic nephropathy. Semin Nephrol 24: 333-344.

12. Nakagawa T, Sato W, Glushakova O, Heinig M, Clarke T, et al. (2007) Diabetic endothelial nitric oxide synthase knockout mice develop advanced diabetic nephropathy. J Am Soc Nephrol 18: 539-550.

13. Boulton AJ (2007) Diabetic neuropathy: classification, measurement and treatment. Curr Opin Endocrinol Diabetes Obes 14: 141-145.

14. Heltianu C, Guja C, Manea SA (2011) Genetic determinants of microvascula complications in type 1 diabetes: Type 1 Diabetes - Pathogenesis, Genetics and Immunotherapy. InTech ISBN 978-953-307-362.

15. Taverna MJ, Elgrably F, Selmi H, Selam JL, Slama G (2005) The T-786C and C774T endothelial nitric oxide synthase gene polymorphisms independently affect the onset pattern of severe diabetic retinopathy. Nitric Oxide 13: 88-92.

16. Costacou T, Chang Y, Ferrell RE, Orchard TJ (2006) Identifying genetic susceptibilities to diabetes-related complications among individuals at low risk of complications: An application of tree-structured survival analysis. Am J Epidemiol 164: 862-872.

17. Brouet A, Sonveaux P, Dessy C, Balligand JL, Feron O (2001) Hsp90 ensures the transition from the early $\mathrm{Ca} 2+-$ dependent to the late phosphorylationdependent activation of the endothelial nitric-oxide synthase in vascular endothelial growth factor-exposed endothelial cells. J Biol Chem 276: 3266332669.

18. Zotova EV, Voron'ko OE, Bursa TR, Galeev IV, Strokov IA, et al. (2005) Polymorphic markers of the NO synthase genes and genetic predisposition to diabetic polyneuropathy in patients with type 1 diabetes mellitus. Mol Bio (Mosk) 39: 224-229.

19. Nosikov VV (2004) Genomics of type I diabetes mellitus and its late complications. Mol Biol (Mosk) 38: 150-164

20. Bazzaz JT, Amoli MM, Pravica V, Chandrasecaran R, Boulton AJ, et al. (2010) eNOS gene polymorphism association with retinopathy in type 1 diabetes Ophthalmic Genet 31: 103-107. 
Citation: Shah VN, Cheema BS, Kohli HS, Sharma R, Khullar M, et al. (2013) Endothelial Nitric Oxide Synthase Gene Polymorphism and the Risk of Diabetic Neuropathy in Asian Indian Patients with Type 2 Diabetes. J Diabetes Metab 4: 243. doi:10.4172/2155-6156.1000243

21. Taverna MJ, Sola A, Guyot-Argenton C, Pacher N, Bruzzo F, et al. (2002) eNOS4 polymorphism of the endothelial nitric oxide synthase predicts risk for severe diabetic retinopathy. Diabet Med 19: 240-245

22. Manea SA, Robciuc A, Guja C, Heltianu C (2011) Identification of gene variants in NOS3, ET-1 and RAS that confer risk and protection against microangiopathy in type 2 diabetic obese subjects. Biochem Biophys Res Commun 407: 486490.

23. Mehrab-Mohseni M, Tabatabaei-Malazy O, Hasani-Ranjbar S, Amiri $P$ Kouroshnia A, et al. (2011) Endothelial nitric oxide synthase VNTR (intron $4 \mathrm{a} / \mathrm{b}$ ) polymorphism association with type 2 diabetes and its chronic complications. Diabetes Res Clin Pract 91: 348-352.

24. Wild S, Roglic G, Green A, Sicree R, King H (2004) Global prevalence of diabetes: estimates for the year 2000 and projections for 2030. Diabetes Care 27: 1047-1053.

25. Sicree R., Shaw J, Zimmet P (2006) Diabetes and impaired glucose tolerance. In Diabetes Atlas (3rdedn), D Gan, International Diabetes Federation, Kortrijik Heule, Belgium.

26. Basu A, Mukherjee N, Roy S, Sengupta S, Banerjee S, et al. (2003) Ethnic India: a genomic view, with special reference to peopling and structure. Genome Res 13: 2277-2290.

27. Shimasaki Y, Yasue H, Yoshimura M, Nakayama M, Kugiyama K, et al. (1998) Association of the missense Glu298Asp variant of the endothelial nitric oxide synthase gene with myocardial infarction. J Am Coll Cardiol 31: 1506-1510.

28. Wang XL, Sim AS, Badenhop RF, McCredie RM, Wilcken DE (1996) A smokingdependent risk of coronary artery disease associated with a polymorphism of the endothelial nitric oxide synthase gene. Nat Med 2: 41-45.

29. Lepoivre M, Chenais B, Yapo A, Lemaire G, Thelander L, et al. (1990) Alterations of ribonucleotide reductase activity following induction of the nitritegenerating pathway in adenocarcinoma cells. J Biol Chem 265: 14143-14149.
30. Hattersley AT, McCarthy MI (2005) What makes a good genetic association study? Lancet 366: 1315-1323.

31. Ahluwalia TS, Ahuja M, Rai TS, Kohli HS, Sud K, et al. (2008) Endothelia nitric oxide synthase gene haplotypes and diabetic nephropathy among Asian Indians. Mol Cell Biochem 314: 9-17.

32. Herrington D (2005) Eliminating the improbable: Sherlock Holmes and standards of evidence in the genomic age. Circulation 112: 2081-2084.

33. Neale BM, Sham PC (2004) The future of association studies: gene-based analysis and replication. Am J Hum Genet 75: 353-362.

34. Ross $R$ (1993) The pathogenesis of atherosclerosis: a perspective for the 1990s. Nature 362: 801-809.

35. Zanchi A, Moczulski DK, Hanna LS, Wantman M, Warram JH, et al. (2000) Risk of advanced diabetic nephropathy in type 1 diabetes is associated with endothelial nitric oxide synthase gene polymorphism. Kidney Int 57: 405-413.

36. Nakayama M, Yasue H, Yoshimura M, Shimasaki Y, Kugiyama K, et al. (1999) T-786-->C mutation in the 5'-flanking region of the endothelial nitric oxide synthase gene is associated with coronary spasm. Circulation 99: 2864-2870.

37. Hibi K, Ishigami T, Tamura K, Mizushima S, Nyui N, et al. (1998) Endothelia nitric oxide synthase gene polymorphism and acute myocardial infarction. Hypertension 32: 521-526.

38. Miyamoto Y, Saito Y, Kajiyama N, Yoshimura M, Shimasaki Y, et al. (1998) Endothelial nitric oxide synthase gene is positively associated with essential hypertension. Hypertension 32: 3-8.

39. Yoshimura T, Yoshimura M, Tabata A, Shimasaki Y, Nakayama M, et al. (2000) Association of the missense Glu298Asp variant of the endothelial nitric oxide synthase gene with severe preeclampsia. J Soc Gynecol Investig 7: 238-241.

40. Cheema BS, kohli HS, Sharma R, Bhansali A, Khullar M (2012) Endothelia nitric oxide synthase gene polymorphism and type 2 diabetic retinopathy among Asian Indians. Acta Diabetol 49: 481-488. 